\title{
Data archive for exploring pedestrian dynamics and its application in dimensioning of facilities for multidirectional streams
}

\author{
Maik Boltes, Stefan Holl, Armin Seyfried \\ Institute for Advanced Simulation, Forschungszentrum Jülich \\ Leo-Brandt-Straße, 52428 Jülich, Germany \\ m.boltes@fz-juelich.de; st.holl@fz-juelich.de; a.seyfried@fz-juelich.de
}

\begin{abstract}
In this paper an overview of an open data archive with data from experiments investigating pedestrian dynamics is presented. As an example of the use of this data the analysis of recently published data about the capacity of crossings is shown.
\end{abstract}

Keywords: data archive, experiments, trajectories, multidirectional, dimensioning

\section{Introduction}

To understand and thereupon to model pedestrian dynamics reliable empirical data is needed. In cooperation with the University of Cologne, Wuppertal and other organizations, we performed 15 experimental series with more than 1500 runs in total and up to 1000 participants in a single run since 2005. We share the data in an open-access archive to ensure transparency and openness and to allow further analysis and the use for model calibration and validation. The data is published as soon as a project is completed.

An example of the use of our data sets is the development of methods dimensioning facilities for multidirectional pedestrian traffic. Based on the trajectories from the research projects Hermes [1] and BaSiGo [2], it was possible to investigate the performance of crossings [3].

\section{Data Archive}

The open access data archive can be found at

$$
\text { http://ped.fz-juelich.de/da }
$$

The data hosted in the archive is the native data used by ourselves. Most of this data include a description or a paper motivating and outlining the experiments, overhead video recordings and (except experiment No. 1 of Tab. 1) trajectories of every single person with a high temporal and spatial resolution [4]. All experiments were performed under laboratory conditions to focus on the impact of a single variable (e.g. geometry, motivation, density) with as few undesired influences as possible. Tab. 1 shows a list of experiments stored in the current data archive. The list includes the place (if no country is specified, the experiment has been conducted in Germany), the scenario, the maximum number of persons in a single run, the number of runs, the project in which context the experiments have been conducted, the first or most important paper to this study and a URL linking to the data (see Sec. 2.2). Fig. 1 shows snapshots of a choice of experiments in the data archive. The list of experiments ends in 2015, but data from studies with people with disabilities in 2017 and about entrance scenarios in 2018 will be added when the associated projects are finished.

The archive is also open to the community. We started to collect data or references to external data from other research groups to create a starting point for experimental studies on pedestrian dynamics: 
Table 1: Experiments in the pedestrian dynamics data archive

\begin{tabular}{|c|c|c|c|c|c|c|c|c|}
\hline No. & Year & Place & Scenario & \# Pers. & \# Runs & Project & Pub. & URL \\
\hline 1 & 2005 & Jülich & single-file & 34 & 6 & & [9] & singleFile \\
\hline 2 & 2005 & Jülich & bottleneck & 60 & 15 & & [11] & bottleneck \\
\hline 3 & 2006 & Düsseldorf & single-file & 70 & 13 & DFG & [10] & singleFile \\
\hline 4 & 2006 & Düsseldorf & bottleneck & 200 & 13 & DFG & [11] & bottleneck \\
\hline 5 & 2009 & Düsseldorf & stairs outside stadium & 200 & 7 & Hermes & [12] & stairsOutside \\
\hline 6 & 2009 & Düsseldorf & mouth hole & 300 & 8 & Hermes & {$[12]$} & mouthHoleUpper \\
\hline 7 & 2009 & Düsseldorf & mouth hole & 300 & 8 & Hermes & [12] & mouthHoleLower \\
\hline 8 & 2009 & Düsseldorf & stairs inside stadium & 300 & 14 & Hermes & [12] & stairsUpper \\
\hline 9 & 2009 & Düsseldorf & stairs inside stadium & 300 & 9 & Hermes & {$[12]$} & stairsLower \\
\hline 10 & 2009 & Düsseldorf & bottleneck & 350 & 5 & Hermes & [13] & bottleneck \\
\hline 11 & 2009 & Düsseldorf & T-junction & 350 & 11 & Hermes & [14] & Tjunction \\
\hline 12 & 2009 & Düsseldorf & corner & 150 & 16 & Hermes & & corner \\
\hline 13 & 2009 & Düsseldorf & corner, free flow & 150 & 3 & Hermes & & cornerFree \\
\hline 14 & 2009 & Düsseldorf & bidirectional flow & 200 & 6 & Hermes & [15] & bidirAsym \\
\hline 15 & 2009 & Düsseldorf & bidirectional flow & 300 & 13 & Hermes & [15] & bidirSym \\
\hline 16 & 2009 & Düsseldorf & bidirectional flow & 300 & 5 & Hermes & [15] & bidirFree \\
\hline 17 & 2009 & Düsseldorf & unidirectional flow & 230 & 24 & Hermes & & unidirClosed \\
\hline 18 & 2009 & Düsseldorf & unidirectional flow & 350 & 31 & Hermes & [16] & unidirOpen \\
\hline 19 & 2012 & Wuppertal & bike, single-file & 33 & 10 & & [17] & bike \\
\hline 20 & 2013 & Düsseldorf & entrance & 319 & 1 & BaSiGo & [18] & entrCorridor \\
\hline 21 & 2013 & Düsseldorf & entrance & 319 & 1 & BaSiGo & [18] & entrSemicircle \\
\hline 22 & 2013 & Düsseldorf & crossing $120^{\circ}$ & 926 & 16 & BaSiGo & [3] & crossing 120 \\
\hline 23 & 2013 & Düsseldorf & crossing $90^{\circ}$ & 603 & 45 & BaSiGo & [3] & crossing 90 \\
\hline 24 & 2013 & Düsseldorf & bidirectional flow & 926 & 16 & BaSiGo & [3] & bidirectional \\
\hline 25 & 2013 & Düsseldorf & unidirectional flow & 916 & 9 & BaSiGo & [3] & unidirectional \\
\hline 26 & 2013 & Düsseldorf & single-file & 58 & 6 & BaSiGo & [19] & singleFile \\
\hline 27 & 2013 & Düsseldorf & social groups & 12 & 18 & BaSiGo & [20] & socialGroups \\
\hline 28 & 2014 & Wuppertal & social groups, pupil & 51 & 132 & DFG & [21] & socialGroups \\
\hline 29 & 2014 & Wuppertal & single-file, pupil & 58 & 105 & DFG & [22] & singleFile \\
\hline 30 & 2015 & Tianshui, China & single-file, age & 71 & 34 & & [23] & singleFile \\
\hline
\end{tabular}

\subsection{Data Collection}

The extraction of individual paths of each participant has been done automatically in most cases with the free software PeTrack [5]. For the extraction of the code marker additional software [6] has been used. Often a manual review and correction of the resulting data has been conducted to increase the quality. The set of trajectories of all pedestrians enables the measurement of quantities like velocity, flow, density and individual distances at any time and position, thus for example lane formation and local densities can be analyzed [7]. Beside the detailed analysis of pedestrians' movement also microscopic models can be designed, calibrated and verified with the data provided [8].

Sometimes the trajectories are enriched with additional global (e.g. distribution of age and gender) or individual (e.g. body size, head orientation) information. The height of the body often was obtained by special marker or 3D sensors like stereo cameras. In experiments where structured marker were used, the head orientation could be extracted. Extensive global individual information could be gathered using code marker and questionnaires as it was realized in experiments No. 19 to 27 of Tab. 1. Here additional information about human factors like gender, weight, age, togetherness, living environment, experiences in dense crowds and much more was gathered. 


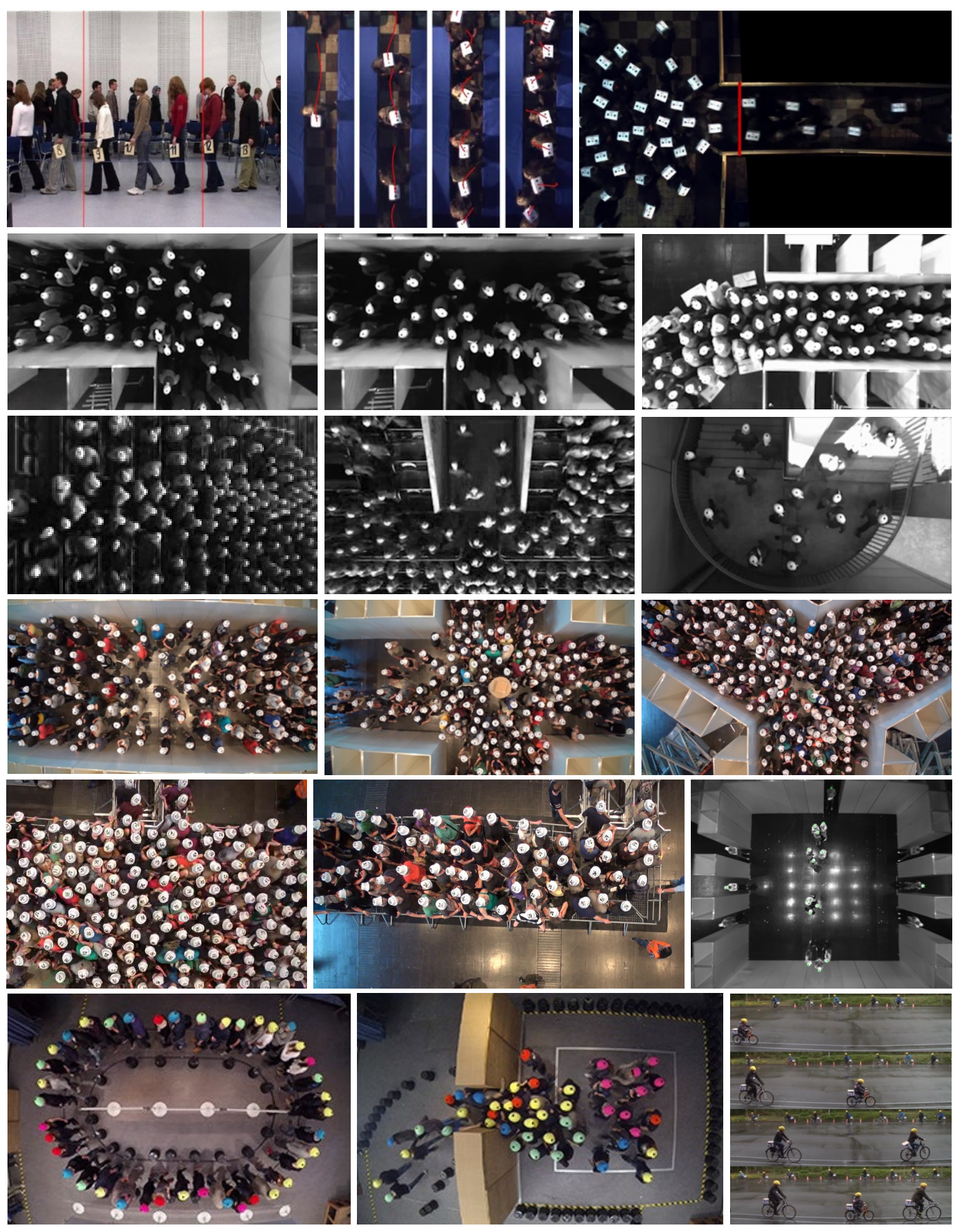

Fig. 1: Snapshots of a choice of experiments for which the data is available in the presented data archive (from left to right, top down): single-file (No. in Tab. 1: 1, 3), bottleneck (4), corner (12), T-junction (11), closed unidirectional flow (17), stairs inside stadium $(8,9)$, mouth hole $(6,7)$, stairs outside stadium (5), bidirectional flow (24), crossings $(22,23)$, entrances $(20,21)$, room with social groups (27), pupil in single-file (29) and at a bottleneck (28), bicycle (19) 


\subsection{Citation}

The use of the data is free, but you are asked to quote the source by citing the corresponding paper of Tab. 1 or if no paper is specified citing [5] describing the collection of the trajectories. To link to a special dataset of the data archive you can use the permanent address build up as follows:

\section{http://ped.fz-juelich.de/da/YearURL}

whereby Year and URL are the corresponding columns of Tab. 1. Thus, for the experiment No. 23 discussed in Sec. 3 the web address is http://ped.fz-juelich.de/da/2013crossing90. More paper and associated videos of the experiments are listed in Tab. 4.3 in [24].

\section{Multidirectional Pedestrian Traffic}

The data obtained by the experiments make it possible to determine characteristic values for the dimensioning of building facilities. The types of pedestrian streams studied in Hermes [1] and BaSiGo [2] are shown in Fig. 2: (a) unidirectional stream, (b) bidirectional stream with two separated lanes, (c) bidirectional stream with multiple lanes, (d) intersecting stream with two orthogonal directions, (e) crossing stream with four orthogonal directions, (f) confluence of two streams at the T-junction, (g) unidirectional stream at a corner, (h) multidirectional stream at triangular intersection.

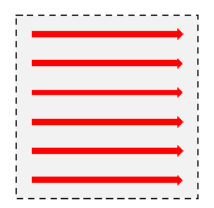

(a)

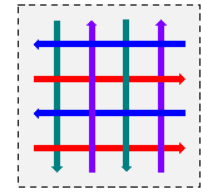

(e)

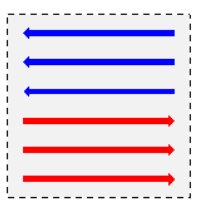

(b)

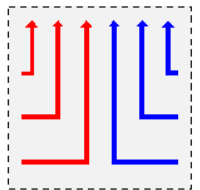

(f)

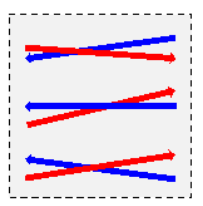

(c)

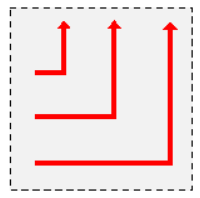

(g)

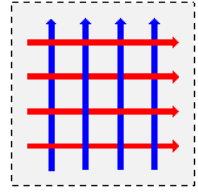

(d)

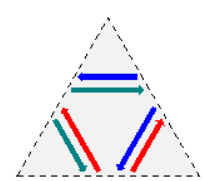

(h)

Fig. 2: Types of streams studied in Hermes and BaSiGo [3]

The results of the intersection analysis (structure type e) are presented below by way of example.

\subsection{Configuration of the experiment for intersection traffic}

For the experiment with multidirectional streams at intersections the crossing was built from 2.5 meter high wooden walls with four entrances arranged at an angle of 90 degrees (see Fig. 3). This spatial structure is referred as CROSSING_90. Each entrance had a clear width of four meters. Above each of the entrances a monitor was mounted. On this it was possible to display roundabout signs as they are known from vehicular traffic. The length of the entrances was $5.00 \mathrm{~m}$ and $5.24 \mathrm{~m}$, respectively, followed by a threemeter-wide exit (labeled "OUT" in Fig. 3) on the left and right. In the center of the core area, a column $(\varnothing=0.6 \mathrm{~m})$ could be positioned.

For the participation a daily fee of 50 Euro was paid. Most of our test persons were students with a mean age of 24.8 years \pm 4.4 years. The ratio between men and women was 55:45. The experiment was carried out on two days in variants A to G. On the first day (variants A, B, C) 319 and on the second day (variants D, E, F, G) 603 test persons participated. 
In variants $\mathrm{A}, \mathrm{B}$ and $\mathrm{C}$, the test persons were evenly distributed over the four entrances, so about 80 people from each side streamed into the intersection. In order not to obstruct the inflows, the persons who had already passed the measuring area were given the opportunity to leave the corridor laterally.

The test persons were asked to use the exit assigned to their feature (even or odd cap number or yellow or red wristband). In variant A there was no column and no signs were displayed on the monitors. In variant $\mathrm{B}$, there was also no column, however, the monitors showed the roundabout sign and the subjects were instructed to follow the instructions on the monitors. In variant $\mathrm{C}$, the pillar in the center of the intersection was additionally set up, the display of the roundabout sign and the instruction to the test persons corresponded to variant $\mathrm{B}$.

In variants $\mathrm{D}$ and $\mathrm{E}$, the test persons were admitted into the intersection only by two entrances arranged at right angles to each other, so about 300 persons streamed in through each of these entrances. In variant $\mathrm{D}$ there was no column and no signs were displayed on the monitors. There was also no column in variant E, but the monitors showed the roundabout sign and the subjects were again asked to pay attention to the information on the monitors.

Variants $\mathrm{F}$ and $\mathrm{G}$ correspond to $\mathrm{C}$ and $\mathrm{B}$ respectively, whereby the width for the inflow has been increased up to $4 \mathrm{~m}$. About 151 people were equally distributed.

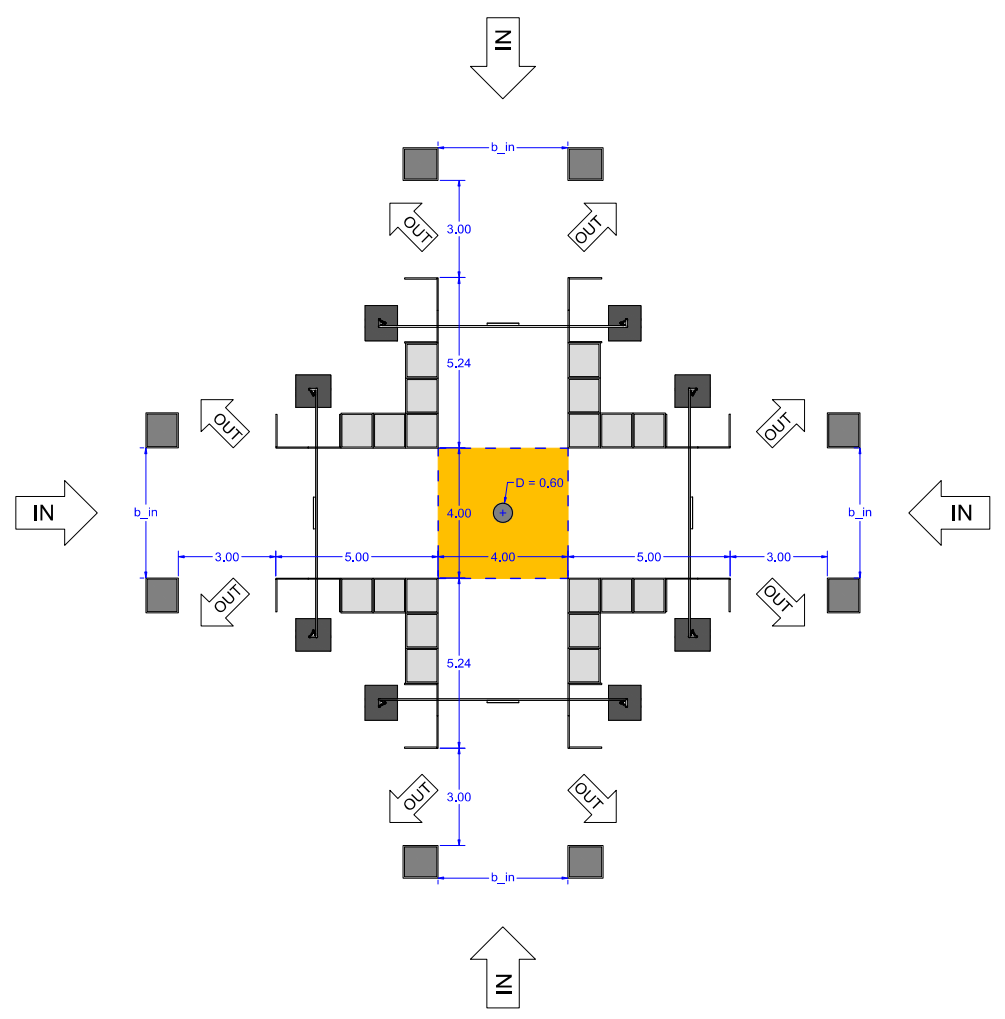

Fig. 3: Setup of experiment CROSSING_90 [3]

\subsection{Analysis of the experiments}

For the intersection with four entrances and multidirectional flows, the BaSiGo experiment CROSSING_90 is analyzed in variants A to G. Fig. 4 shows a picture and Fig. 5 an example of the trajectories for a run with a column in the center of the intersection. 


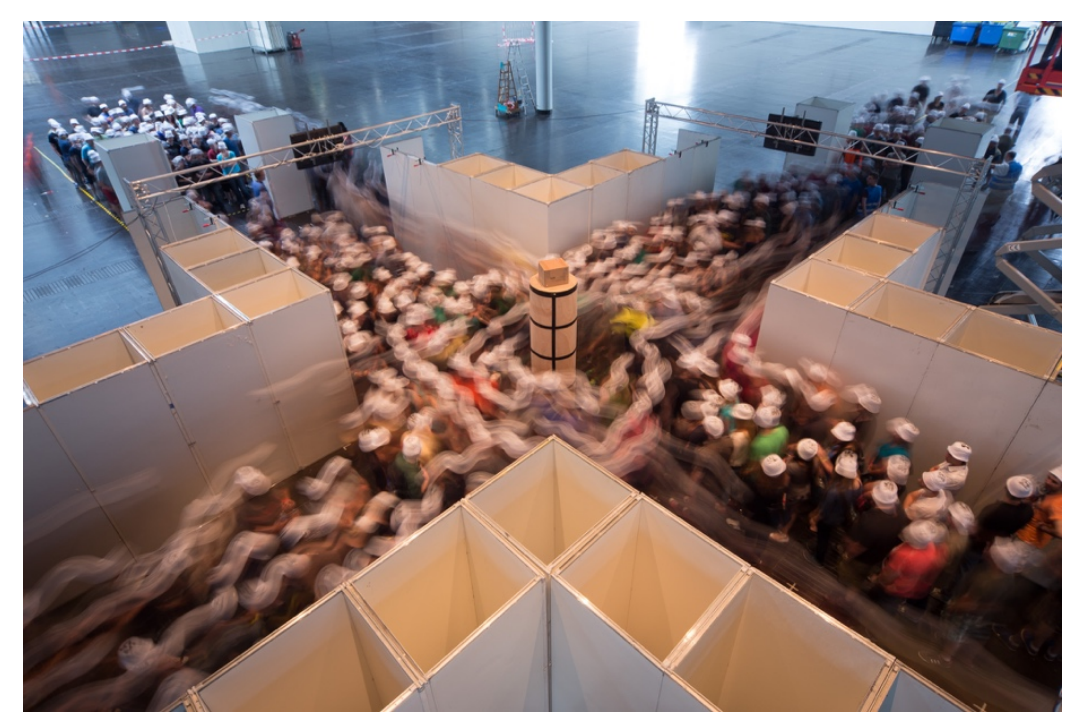

Fig. 4: Execution of experiment CROSSING_90 [3]

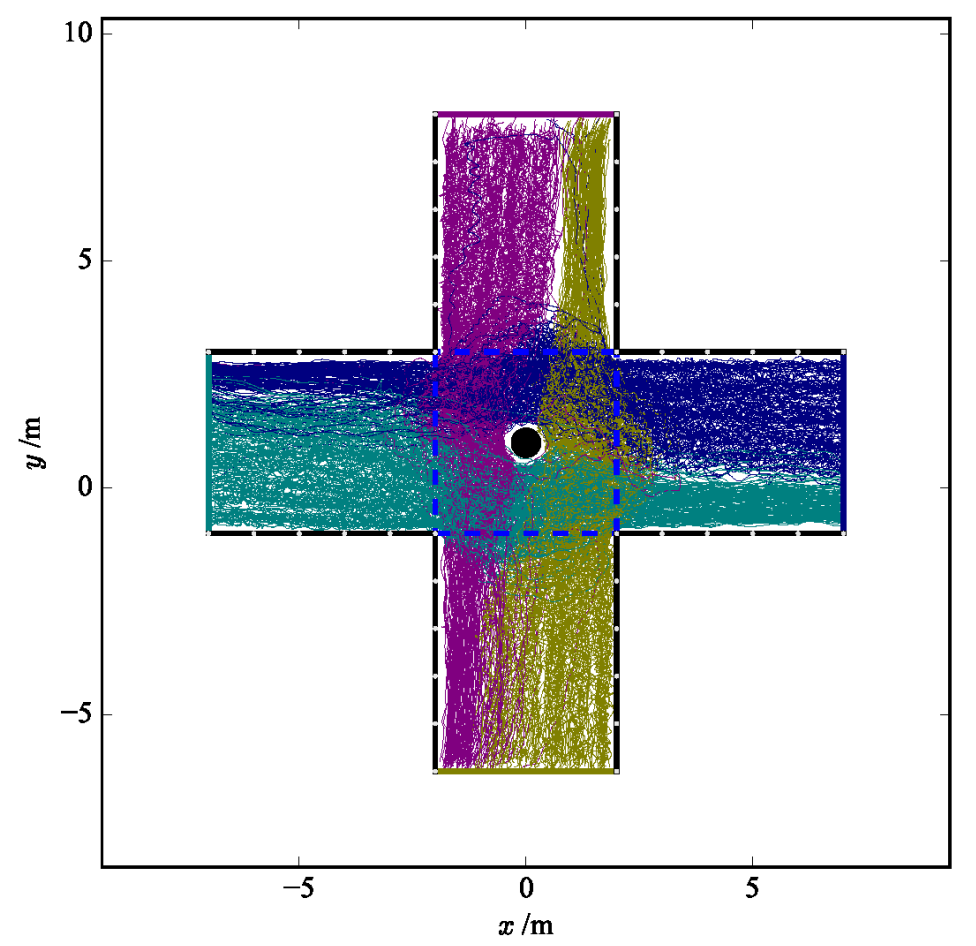

Fig. 5: Trajectories of a single run with four entrances and a column in the center of the intersection [3]

For our analyzes, a measurement method following [25] was used that allows to calculate density $\rho$, flow $J$ and speed $v$ in a multidirectional traffic system as averages over the same place and time [3,26]. Fig. 6 shows the fundamental diagrams $v(\rho)$ and $J_{s}(\rho)$ for the measurement area shown as dashed blue line in Fig. 5. The specific flow $J_{s}$ is calculated as the product of the mean velocity $v$ and the mean density $\rho$. The data show no significant differences in the variants of the experiment. Both for the number of accesses used (two accesses in variants $\mathrm{D}$ and $\mathrm{E}$, four accesses in all other variants) as well as for the column in the center of the crossing (variants $\mathrm{C}$ and $\mathrm{F}$ ) there is no significant influence on the fundamental diagram in the 
density regime covered in the experiments. Noteworthy are the high densities of up to seven people per square meter.

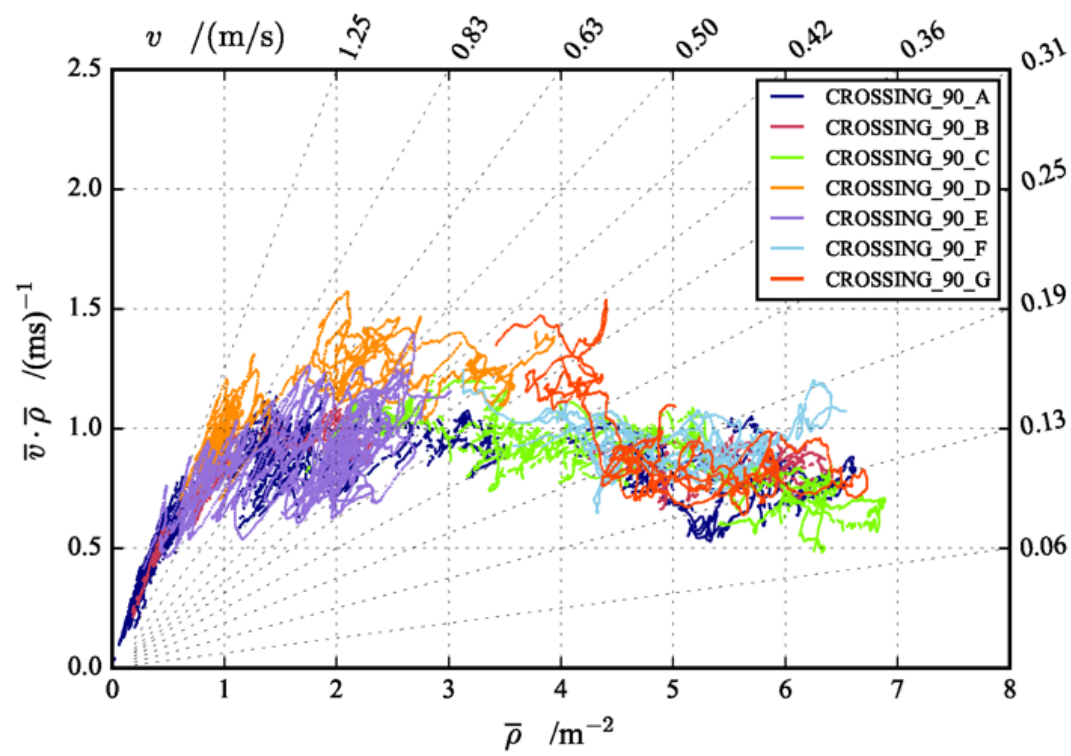

Fig. 6: Fundamental diagram for crossing pedestrian traffic $[3,26]$

\section{Acknowledgements}

Most of the experiments have been performed within projects funded by the German Research Foundation (DFG-Grant No. KL 1873/1-1, SE 1789/1-1 or No. SCHA 636/9-1) or Federal Ministry of Education and Research (BMBF) (e.g. Hermes, BaSiGo). For the design and performance of the experiments we collaborated with various universities, especially the Universities of Wuppertal, Cologne and Siegen.

\section{References}

[1] S. Holl, A. Seyfried, "Hermes - an evacuation assistant for mass events", in inSiDe, vol. 7, 2009, pp. 60-61.

[2] S. Holl, M. Boltes, A. Seyfried, "Level of Safety Concept for Major Events", in Proceedings of Traffic and Granular Flow 2015, 2016, pp. 337-344.

[3] S. Holl, "Methoden für die Bemessung der Leistungsfähigkeit multidirektional genutzter Fußverkehrsanlagen“, in Schriften des Forschungszentrums Jülich, IAS Series, vol. 32, 2016.

[4] M. Boltes, S. Holl, A. Tordeux, A. Seyfried, A. Schadschneider, U. Lang, "Influences of Extraction Techniques on the Quality of Measured Quantities of Pedestrian Characteristics", in Proceedings of Pedestrian and Evacuation Dynamics 2016, Collective Dynamics, 2016, pp. 540-547.

[5] M. Boltes, A. Seyfried, "Collecting pedestrian trajectories", Neurocomputing, vol. 100, 2013, pp. 127-133.

[6] W. Mehner, M. Boltes, A. Seyfried, "Methodology for generating individualized trajectories from experiments", in Proceedings of Traffic and Granular Flow 2015, 2015, pp. 3-10.

[7] J. Liddle, A. Seyfried, B. Steffen, "Analysis of bottleneck motion using Voronoi diagrams", in Proceedings of Pedestrian and Evacuation Dynamics 2010, 2011, pp. 833-836.

[8] B. Steffen, A. Seyfried, M. Boltes, "Reliability issues in the microscopic modeling of pedestrian movement", in Mathematical results in Quantum Physics, 2011, pp. 254-259.

[9] A. Seyfried, B. Steffen, W. Klingsch, M. Boltes, "The Fundamental Diagram of Pedestrian Movement Revisited”, in J. Stat. Mech.: Theory Exp. P10002, 2005. 
[10] A. Seyfried, M. Boltes, J. Kähler, W. Klingsch, A. Portz, T. Rupprecht, A. Schadschneider, B. Steffen, A. Winkens, "Enhanced empirical data for the fundamental diagram and the flow through bottlenecks", in Proceedings of Pedestrian and Evacuation Dynamics 2008, 2010, pp. 145-156.

[11] A. Seyfried, T. Rupprecht, O. Passon, B. Steffen, W. Klingsch, M. Boltes, "New insights into pedestrian flow through bottlenecks", in Transportation Science, 43, 2009, pp. 395-406.

[12] S. Burghardt, A. Seyfried, W. Klingsch, "Performance of stairs - Fundamental diagram and topographical measurements", in Transportation research, C 37, 2013, pp. 268-278.

[13] L. Weichen, A. Seyfried, J. Zhang, M. Boltes, X. Zheng, Y. Zhao, "Experimental Study on Pedestrian Flow through Wide Bottleneck" in Transportation Research Procedia, vol. 2, 2014, pp. 26-22.

[14] M. Boltes, J. Zhang, A. Seyfried, B. Steffen, "T-junction: Experiments, trajectory collection, and analysis", in Proceedings of International Conference on Computer Vision 2011 (Workshops), 2012, pp. 158-165.

[15] J. Zhang, W. Klingsch, A. Schadschneider, A. Seyfried, "Ordering in bidirectional pedestrian flows and its influence on the fundamental diagram", in Journal of statistical mechanics: theory and experiment, P02002, 2012.

[16] J. Zhang, W. Klingsch, A. Schadschneider, A. Seyfried, "Transitions in pedestrian fundamental diagrams of straight corridors and T-junctions", in Journal of Statistical Mechanics: Theory and Experiment, P06004, 2011.

[17] J. Zhang, W.Mehner, S.Holl, M.Boltes, E.Andresen, A.Schadschneider, A.Seyfried, "Universal flowdensity relation of single-file bicycle, pedestrian and car motion", in Physics Letters A, vol. 378, issue 44, 2014, pp. 3274-3277.

[18] A. Sieben, J. Schumann, A. Seyfried, "Collective phenomena in crowds - Where pedestrian dynamics need social psychology", in PLOS one, 2017.

[19] V. Ziemer, A. Seyfried, A. Schadschneider, "Congestion Dynamics in Pedestrian Single-File Motion", in Proceedings of Traffic and Granular Flow 2015, 2016, pp. 89-96.

[20] N. W. F. Bode, S. Holl, W. Mehner, A. Seyfried, "Disentangling the Impact of Social Groups on Response Times and Movement Dynamics in Evacuations", in PLOS one, 2015.

[21] C. von Krüchten, F. Müller, A. Svachiy, O. Wohak, A. Schadschneider, "Empirical Study of the Influence of Social Groups in Evacuation Scenarios", in Proceedings of Traffic and Granular Flow 2015, 2016, pp. 65-72.

[22] J. Wang, M. Boltes, A. Seyfried, J. Zhang, V. Ziemer, W. Weng, "Linking pedestrian flow characteristics with stepping locomotion", in Physica / A 500, 2018, pp. 106-120.

[23] S. Cao, J. Zhang, D. Salden, J. Ma, C. Shi, R. Zhang, "Pedestrian dynamics in single-file movement of crowd with different age compositions", in Phys. Rev. E 94, 012312, 2016.

[24] M. Boltes, "Automatische Erfassung präziser Trajektorien in Personenströmen hoher Dichte“, in Schriften des Forschungszentrums Jülich, IAS Series, vol. 27, 2015.

[25] L. C. Edie, "Discussion of traffic stream measurements and definitions", in Proceedings of the Second International Symposium on the Theory of Traffic Flow, 1965, pp. 139-154.

[26] S. Cao, A. Seyfried, J. Zhang, S. Holl, W. Song, "Fundamental diagrams for multidirectional pedestrian flows “, in Journal of Statistical Mechanics: Theory and Experiment, P033404, 2017. 